\title{
WP-(8)-13
}

\section{A Full-Vectorial Multidomain Spectral Collocation Modal Analysis of Dielectric Optical Waveguides}

\author{
Chia-Chien Huang*, Chia-Chih Huang**, and Jaw-Yen Yang*
}

${ }^{*}$ Computational Electromagnetics and Plasma Lab., Institute of Applied Mechanics,

$$
\text { National Taiwan University }
$$

No. 1, Sec. 4, Roosevelt Road, Taipei 106, Taiwan, ROC

Tel: 886-2-3366-5636 Fax: +886-2-2363-9290 Email: yangjy@spring.iam.ntu.edu.tw

**Department of Electrical Engineering and Graduate Institute of Electro-Optical

Engineering, National Taiwan University

Abstract-A novel solution method using efficient multidomain spectral collocation method in term of transverse magnetic fields is proposed for vector modal analysis of dielectric optical waveguides with discontinuous refractive index profile. The computational domain is divided into a few subdomains at the interfaces of different materials. Each one can be expanded by a suitable set of orthogonal basis functions and patched by considering the interfacial boundary conditions.

Modal analysis is an essential consideration for analyzing the propagation characteristics of optical waveguides. Recently, spectral collocation method with usual single domain is proposed [1]. However, the oscillatory result and poor convergence are encountered while the discontinuous RIP's exist [1]. Moreover, the multidomain spectral collocation method which divides the whole interest domain into a few subdomains at the interfaces of different materials is proposed to tackle the problems. Furthermore, the interfacial boundary conditions of the transverse magnetic fields are utilized to patch these subdomains. To check the accuracy of the present method, a rectangular channel waveguide is considered. The dispersion relations of the lowest order of $H^{y}$ and $H^{x}$ modes obtained by both Goell [2] and by the present method are shown in Fig. 1. We can observe from Fig. 1 that our results show excellent agreement with Goell's solutions. The present method is expected to further apply to more complicated optical devices of great interest.

[1] S. Banerjee and A. Sharma, "Propagation characteristics of optical waveguiding structure by direct solution of the Helmholtz equation for total fields," J.Opt. Soc. Am. A 6, pp. 1884-1894, (1989).

[2] J. E. Goell, "A circular harmonic computer analysis of rectangular dielectric waveguides," Bell Syst. Tech. $J ., 48$, pp. 2133-2160, (1969).

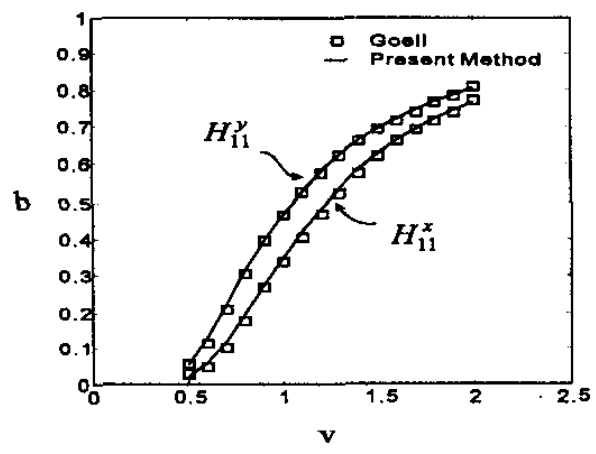

Fig. 1 Normalized propagation constant $b$ of the $H_{11}^{y}$ and $H_{11}^{x}$ modes vs. the normalized frequency v. 